\title{
Prevention and Treatment of Multiple Osteoporotic Compression Fracture
}

\author{
Ye-Soo Park, Hee-Soo Kim \\ Department of Orthopaedic Surgery, Guri Hospital, Hanyang University College of Medicine, Guri, Korea
}

Korea's demographic profile is undergoing tremendous change as the country rapidly ages at one of the fastest rates in the world. Indeed, the country is expected to become an "aged society" in 2018 when the proportion of elderly is estimated to reach $14.3 \%$ of the total population. With the notable increase in the number of elderly individuals, the incidence of osteoporotic fractures will also likely increase. Osteoporosis is a systemic musculoskeletal disease that is characterized by the decreased bone quantity and the abnormalities of the microstructures. There are both conservative and surgical treatment modalities for the fracture: conservative treatments include pharmacological treatments and orthosis; surgical treatments include vertebroplasty, kyphoplasty, and reconstructive surgery. Clinicians should consider the severity of osteoporosis, the concurrent osteoporotic fracture, the age and sex of the patient, and the underlying diseases in making a patient-tailored prescription.

Keywords: Osteoporotic compression fracture; Conservative treatment; Vertebroplasty; Kyphoplasty; Reconstructive surgical procedures

\section{Introduction}

The World Health Organization defines osteoporosis as a systemic musculoskeletal disease characterized by decreased bone quantity and microstructural abnormalities. This disease causes bones to become fragile and vulnerable to fracture. Thus, osteoporosis is characterized by decreased bone strength: Bone quality accounts for $70 \%$ of bone strength; bone quantity accounts for 30\% [1]. Bone quality is dependent on structural changes in bony structure, while bone quantity is dependent on bone mineral density (BMD). The incidence of bone fractures increases with age and is unrelated to BMD [2]. Factors other than BMD are involved in the occurrence of bone fractures, and they include bone fracture. Recent studies have shown that the Korean population is aging more rapidly than any other country in the world. According to Statistics Korea, a government statistical agency, Korea became an "Aging Society" in 2000 when the proportion of elderly people reached $7.2 \%$ of the total population. Korea will become an aged society in 2018 when the proportion of elderly people is estimated to reach $14.3 \%$ of the total population; it will become a super-aged society in 2026 when this proportion is estimated to reach $20.8 \%$ [3]. As the number of elderly people increases, the incidence of osteoporotic fractures will also likely increase. The resulting increase in medical expenses could cause serious socio-economic losses [3].

\section{Discussion}

Osteoporotic fractures have the following characteristics: 1) The age of patients with osteoporotic fractures is relatively high. In addition, these patients commonly have comor-

Received Oct 28, 2013; Revised Nov 15, 2013; Accepted Nov 16, 2013

Corresponding author: Ye-Soo Park

Department of Orthopaedic Surgery, Hanyang University Guri Hospital, Hanyang University College of Medicine,

153 Gyeongchun-ro, Guri 471-701, Korea

Tel: +82-31-560-2316, Fax: +82-31-557-8781, E-mail: hyparkys@hanyang.ac.kr 
bidities such as hypertension, diabetes, and myocardial infarction; 2) Careful observation is needed for patients with markedly decreased preoperative activity and those with psychiatric diseases such as dementia; 3 ) Reduced bone strength increases the risk of fixation loss; 4) Reduced BMD increases the risk of fracture even when patients experience lower-intensity external force; 5) A fracture may not be detected immediately because osteoporotic patients are vulnerable to occult and insufficiency fractures, in which the fracture lines are almost invisible $[4,5]$;

In addition, surgery cannot be performed on some patients with severe medical conditions because these patients cannot be anesthetized. Patients with osteoporosis and concurrent fractures are also at increased risk of developing gait disturbance and muscle weaknessconditions that increase the risk of mortality and morbidity. These changes may also be accompanied by socioeconomic losses.

Unlike more general types of vertebral fractures, osteoporotic fractures are considered pathological fractures. The treatment modalities used for other types of fractures are also attempted for osteoporotic fractures. Unless appropriately treated, osteoporotic fractures can lead to multiple vertebral fractures, especially in patients with secondary osteoporosis. To prevent multiple osteoporotic vertebral fractures, early prevention should be considered. This should be accompanied by more aggressive treatment in patients with fractures.

Left untreated, osteoporosis causes osteoporotic fractures. Osteoporosis can be classified into postmenopausal osteoporosis, in which estrogen secretion is decreased due to menopause, primary osteoporosis due to aging (also known as senile osteoporosis), and secondary osteoporosis due to causes such as endocrine disorders, steroid use, hematologic tumors, impaired bone matrix synthesis, nutritional insufficiency, and vitamin D metabolic disorder.

Vertebral compression fractures frequently occur in patients 60 years or older who have postmenopausal osteoporosis due to estrogen deficiency. In patients with autoimmune disease, however, osteoporotic bone loss is associated with the period of use and the dose of glucocorticoids [6]. Furthermore, many cases of multiple vertebral fractures are due to secondary osteoporosis. To date, however, no studies have examined the epidemiology and treatment modalities for vertebral fractures due to secondary osteoporosis in Korean patients.

At present, glucocorticoids are frequently used to treat various diseases in a clinical setting over a short- or longterm period. Glucocorticoids affect various phases of the differentiation and calcium metabolism of osteocytes. Long-term use of glucocorticoids typically causes biphasic bone loss. For the first several months of use, bone quantity rapidly decreases by $10 \%$ to $15 \%$; thereafter, it decreases by $2 \%$ to $5 \%$ annually [7]. Osteoporosis due to glucocorticoid use is characterized by decreased bone formation and increased bone resorption, which eventually leads to more rapid loss of bone mass. Loss of bone mass occurs mainly in areas with abundant trabecular bone such as the vertebrae or ribs (Fig. 1). Glucocorticoids have a strong impact on the axial skeleton and proximal femur. The earliest osteoporotic lesions are usually observed on lumbar lateral X-rays. It has been reported that more than $50 \%$ of patients experience bone loss and 25\% of patients experience fracture after taking prednisolone $7.5 \mathrm{mg}$ daily for more than three months [6]. Choi et al. [8] observed no bone fracture in patients who received glucocorticoids for less than one year; however, bone fractures occurred at an incidence of $33 \%$ in patients who received glucocorticoids for 3 to 5 years and recorded an incidence of $56 \%$ in patients who received it for more than five years. Their study also examined the incidence of bone fracture among patients of different ages who used glucocorticoids for more than five years. They found that the incidence of bone fracture was $37 \%$ in those between 50 and 59 years and $88 \%$ in those 70 years or older. Thus, bone fractures appear to be more common in older patients with longer exposure to glucocorticoids [8]. The prevention and treatment of osteoporotic fractures in patients who have received long-term administration of glucocorticoids poses challenges for clinicians. Currently, there is no management protocol for these patients. Moreover, the progression of vertebral compression fractures increase during a short-term period. Currently, it is difficult to predict when a bone fracture will occur, and it is challenging to determine the scope of treatment that will be required once it occurs. Systematic, in-depth, multi-disciplinary approaches are needed in which doctors evaluate both the fracture site and the systemic status of patients in collaboration with other specialty areas such as internal medicine, general surgery, and anesthesiology.

The treatment goals for osteoporosis due to glucocorticoid use are as follows: 1) To maintain current bone quantity and to prevent the occurrence of further bone resorption; 2) To reduce pain associated with bone frac- 
ture; 3) To maintain and increase muscle strength; 4) To improve living habits;

The methods for preventing osteoporosis include skeletal loading by physical activity, dietary calcium and phosphate intake, and abstinence from smoking and excessive drinking. In addition, sodium restriction, increased intestinal absorption of calcium by thiazide diuretics, and decreased excretion of calcium through urine are also helpful for preventing osteoporosis. Pharmacological treatments include various types of drugs. The choice of drugs varies in relation to the severity of osteoporosis, the presence of concurrent osteoporotic fractures, the age and sex of patients, and the underlying diseases leading to osteoporosis. These factors should be considered when deciding a patient-tailored treatment plan.

Both conservative and surgical treatments can be used to treat fractures. Conservative treatments include drugs and orthosis. Surgical treatments include vertebroplasty, kyphoplasty, and reconstructive surgery.

\section{Pharmacological Treatments for the Prevention of a Multiple Bone Fracture (Table 1)}

\section{Calcium and vitamin $\mathrm{D}$}

Both calcium and vitamin D are critical for the preven- tion and treatment of osteoporosis. If there is a deficiency in calcium and vitamin $\mathrm{D}$, serum levels of parathyroid hormone (PTH) will increase to maintain a consistent level of calcium. In patients aged 50 years or older, the daily recommended dose of calcium is $1,000-1,200 \mathrm{mg}$, although the daily dose for maximal absorption should be lower than $500 \mathrm{mg}$. There do not appear to be further benefits to increasing the calcium dose above the recommended daily intake. In addition, excessive calcium supplementation increases the risks of developing nephrolithiasis. Special attention should be paid to elderly patients, or those with renal failure, because these patients are at increased risk of developing cardiovascular disease (such as myocardial infarction) due to excessive calcium supplementation. Calcium monotherapy is not recommended for patients with severe osteoporosis accompanied by vertebral fractures. These patients should receive a concomitant medication of calcium and antiosteoporotic drugs. Moreover, vitamin D deficiency causes sarcopenia and muscle weakness $[9,10]$ because $1,25(\mathrm{OH}) 2 \mathrm{D}$ binds to a specific receptor in the nucleus of myofibroblasts and thereby promotes protein synthesis and growth.

Vitamin D has been known to play a key role in increasing muscle strength, improving balance, and reduc-
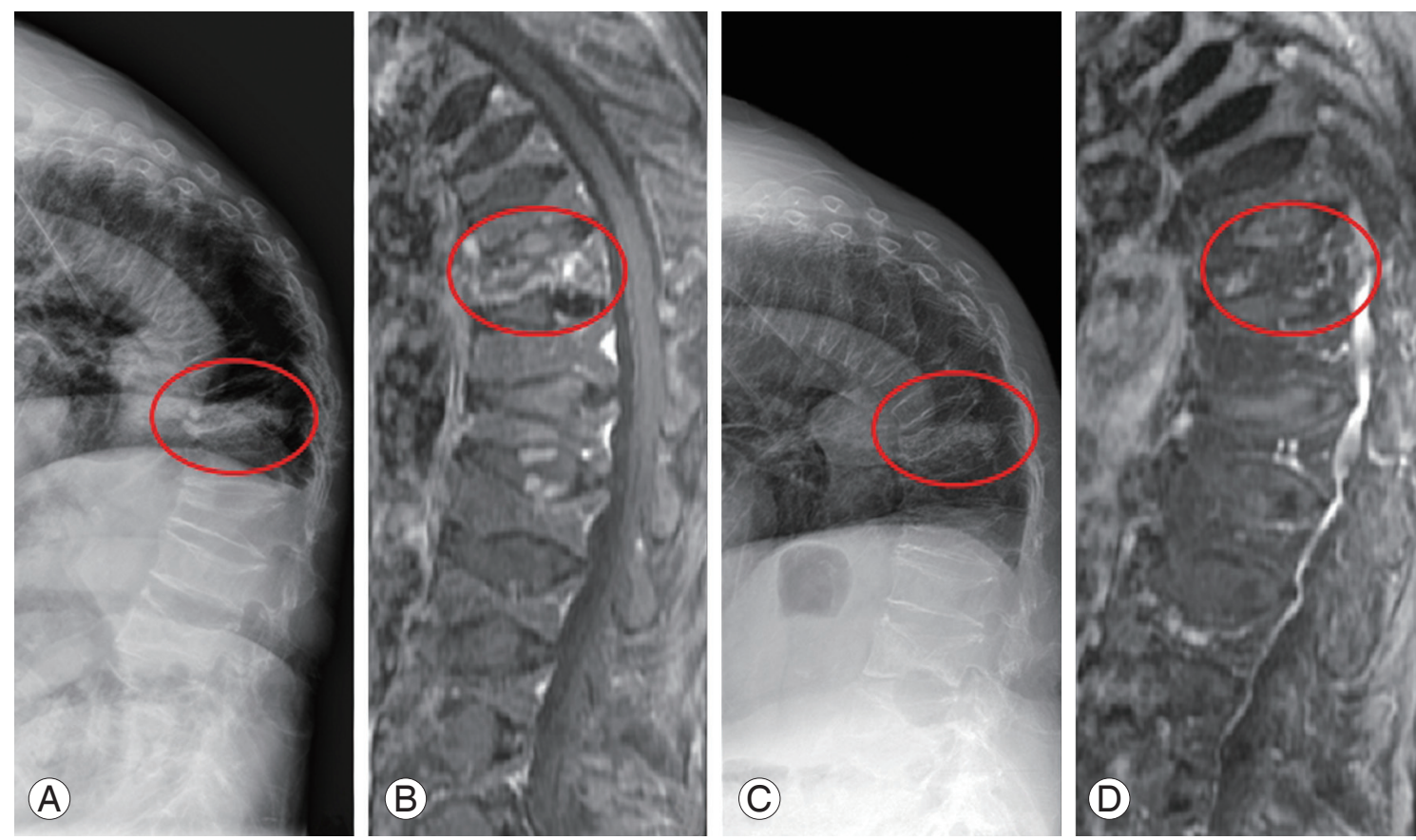

Fig. 1. (A, B) A 74-year-old female's simple radiograph and magnetic resonance imaging (MRI) demonstrating acute osteoporotic compression fracture in T12, multiple old compression fractures, and resultant kyphosis. (C, D) Simple lateral X-ray and MRI at 3 months after of parathyroid hormone injection show the consolidation of the fracture site. 
Table 1. Recommended pharmacologic agents to treat osteoporosis and reduce fracture risk

\begin{tabular}{|c|c|c|c|}
\hline & Vertebral fracture & Hip fracture & Non-vertebral fracture \\
\hline \multicolumn{4}{|l|}{ Bisphosphonate } \\
\hline Alendronate & A & A & A \\
\hline Risedronate & A & A & A \\
\hline Etidronate & A & C & C \\
\hline Hormone replacement therapy & $A$ & A & $A$ \\
\hline SERM (Raloxifene) & A & C & C \\
\hline Calcitonin, intranasal & A & C & C \\
\hline Teriparatide & A & - & A \\
\hline \multicolumn{4}{|c|}{ Calcium and vitamin D preparations } \\
\hline Vitamin D monotherapy & C & C & C \\
\hline Calcium monotherapy & $\mathrm{B}$ & C & C \\
\hline Vitamin D plus calcium & C & A & A \\
\hline
\end{tabular}

A, convincing evidence of antifracture efficacy; $C$, ineffective or insufficient of efficacy; SERM, selective estrogen receptor modulator; B, inconsistent results.

ing the occurrence of fall injuries [11]. Moreover, vitamin D deficiency leads to the absorption of just $10 \%$ to $15 \%$ of the total calcium of a patient's dietary intake. When vitamin D deficiency is not an issue, up to $30 \%$ to $40 \%$ of total dietary calcium is absorbed.

\section{Bisphosphonates}

The treatment methods for osteoporosis can be classified into two categories: those that increase bone mineral density and those that improve bone quality. In the field of orthopedics, calcium and vitamin D are currently used for the treatment of approximately $30 \%$ of patients with osteoporosis. Bisphosphonates are used for the treatment of approximately $55 \%$ of patients. The clinical use of bisphosphonate was first attempted in 1960. Since then, various types of bisphosphonates have become commercially available. Their mode of action is based on the inhibition of osteoclastic bone resorption, the promotion of osteoclastic apoptosis, the suppression of differentiation of osteoclasts from hematopoietic stem cells, the promotion of proliferation and differentiation of proosteoclasts, and the stimulation of osteoblastic synthesis of osteoprotegerin, which suppresses bone resorption. Bisphosphonates can be taken orally or through injection. Oral formulations include alendronate, risedronate, and ibandronate, which cannot be used in patients with esophageal and GI diseases and those confined to all-day bed rest. Normal, healthy individuals often choose to discontinue the use of oral formulations of bisphosphonate because of the inconvenience of administration. Oral formulations of bisphosphonate have produced excellent treatment outcomes. However, some patients develop adverse effects due to long-term use and some develop GI disturbances. Intravenous injection of bisphosphonate can be used at a 3-month interval (ibadronate) or 1-year interval (zoledronate). Injection of bisphosphonate is associated with higher patient compliance than oral administration. It is very useful in patients, especially elderly patients, who are taking other drugs for the treatment of comorbidities such as hypertension and diabetes mellitus. Approximately $0.6 \%$ to $1 \%$ of oral formulations are absorbed after administration, but the bioavailability of bisphosphonate after injection reaches up to $100 \%$. Moreover, injection with bisphosphonate produces almost no GI adverse effects.

\section{Selective estrogen receptor modulator}

Representative drugs that improve bone quality include selective estrogen receptor agonists and selective estrogen receptor modulators (SERMs). The SERMs function as agonists for bone tissue and antagonists for the breast and uterus. Little is known, however, about their modes of action. Raloxifene and Bazedoxifene are the SERMs currently available commercially. A review of the extant literature 
suggests that SERMs are less effective in increasing BMD than bisphosphonates and that they are not effective in preventing the occurrence of non-vertebral fracture.

\section{Parathyroid hormone}

Parathyroid hormone (PTH) is a peptide hormone composed of 84 amino acids that plays a key role in regulating calcium homeostasis. As observed in patients with hyperparathyroidism, a persistent increase in serum PTH levels leads to bone loss. However, an intermittent, low-dose treatment with PTH can increase bone quantity. PTH is involved in the activation of osteoblasts and the stimulation of osteoclasts. Thus, it produces an anabolic window period in which the level of bone formation is at its highest. These phenomena have been confirmed in clinical studies examining alterations in bone-turnover markers. After treatment with parathyroid hormone, serum levels of osteoblastic markers are elevated; this is followed by elevation of the serum levels of osteoclastic markers approximately three months later. Thus, total bone quantity is increased. From a physiologic perspective, PTH is involved in the direct stimulation of osteoblast proliferation, accompanied by the suppression of osteoblast apoptosis. It indirectly stimulates secretion of growth factors and suppresses secretion of sclerostin, which inhibits the activity of osteoblasts. Thus, PTH is involved in the promotion of osteoblastic activity [7]. Its clinical use was first approved by the US Food and Drug Administration. Currently, it is the only drug available that promotes osteoblastic activity. PTH (I-34) appears to reduce the occurrence of bone fracture. In addition, its ability to increase BMD and decrease the occurrence of bone fracture have been demonstrated in postmenopausal women and men with steroid-induced osteoporosis $[12,13]$. Furthermore, a comparative study between PTH and bisphosphonate confirmed that PTH had excellent efficacy in increasing BMD [14].

PTH is suitable for patients with multiple osteoporotic vertebral fractures who are not candidates for surgery. However, its high cost can be a deterrent to some patients. Unless patients receive continuous treatment after a maximum treatment period of two years, the osteoporosis rapidly worsens and may lead to osteoporotic fractures. It can therefore be inferred that continuous pharmacological treatments are effective in the prevention of multiple bone fractures.
One of the most common adverse effects of PTH is hypercalcemia, which occurs in $1 \%$ to $3 \%$ of total patients. In these patients, clinicians should first consider lowering serum levels of calcium or vitamin D. In patients who are refractory to these methods, however, PTH should be administered at 2-day intervals to resolve hypercalcemia [15]. Serum calcium levels should be measured one month after treatment with PTH. Some patients who take PTH will experience transiently elevated serum uric acid levels, although most of these cases are not clinically significant. Moreover, PTH can produce adverse effects, such as dizziness and nausea due to orthostatic hypotension and pain in the lower extremities. The following patients are not candidates for PTH therapy: 1) Patients with Paget's disease; 2) Patients with unexplained elevations of alkaline phosphatase; 3) Pediatric patients; 4) Patients who are at increased risk of developing osteosarcoma, such as those who have had radiotherapy for bone lesions; 5) Patients with cancer with bone metastases; 6) Patients with malignant bone tumors; 7) Patients with metabolic bone diseases other than osteoporosis; 8) Patients with hypercalcemia; 9) Patients who are pregnant or breastfeeding;

In addition, special attention should be paid to patients with a past or current history of ureteral stone and those taking digitalis. Even in indicated patients, the period of PTH therapy should be less than two years. This is based on an animal experiment showing that osteosarcoma occurs after approximately two years of treatment with PTH in rats (Fig. 1) [16]. Moreover, PTH therapy cannot be performed for patients with creatinine clearance of $<30 \mathrm{~mL} / \mathrm{min}$.

\section{Surgical Treatments}

Persistent pain, due to instability, is one of the most common indications for surgery in patients with osteoporotic vertebral fractures. Although rare, some patients present with neurological symptoms. These patients may also be treated with nerve decompression, internal fixation, and bone fusion. It is problematic, however, that the rigidity of internal fixation is relatively lower than in healthy populations due to osteoporosis. Recently, there has been increased interest in vertebroplasty: In which a needle is inserted in the fractured vertebral body and substances (such as bone cement) are infused, and balloon kyphoplasty, in which the vertebral depression is reconstructed with a balloon before infusion with a substance such as 
bone cement. Vertebroplasty is performed on patients who present with persistent severe pain despite appropriate conservative treatments for 2 to 3 weeks after fracture occurrence. Patients with blood coagulation disorders or fractures accompanied by neurological symptoms are not candidates for vertebroplasty. In patients with stroke, dementia, Cushing's syndrome, renal failure, severe pain, and those whose systemic status might be worsened due to long-term conservative treatments, however, early treatments can be performed. It is generally accepted that the incidence of complications is increased when surgery is performed on more than three vertebral bodies. Little is known about the mechanisms by which pain is relieved after vertebroplasty with bone cement. It has been suggested, however, that these mechanisms are related to mechanical stability and destruction of the nerve terminal due to the exothermic reaction innervating adjacent soft tissue. One of the most common complications of vertebroplasty is the leakage of bone cement: Bone cement may leak into the vertebral canal, the adjacent intervertebral disc, and the veins and soft tissue around the vertebral body during vertebroplasty. One of the most serious complications of bone cement leakage is paralysis due to nerve compression. To avoid this complication, bone cement with the lowest viscosity cannot be infused. Moreover, infusion of the bone cement should be stopped before it reaches the posterior cortical bone on the image intensifier. Other complications of vertebroplasty include fracture of the adjacent vertebral body and vertebral body recollapse. Vertebroplasty may be effective in reducing pain and increasing mechanical strength (Fig. 2). It can prevent aggravation of the collapse, but it cannot effectively improve the kyphotic deformity. Moreover, it is problematic that bone cement leaks out of the vertebral
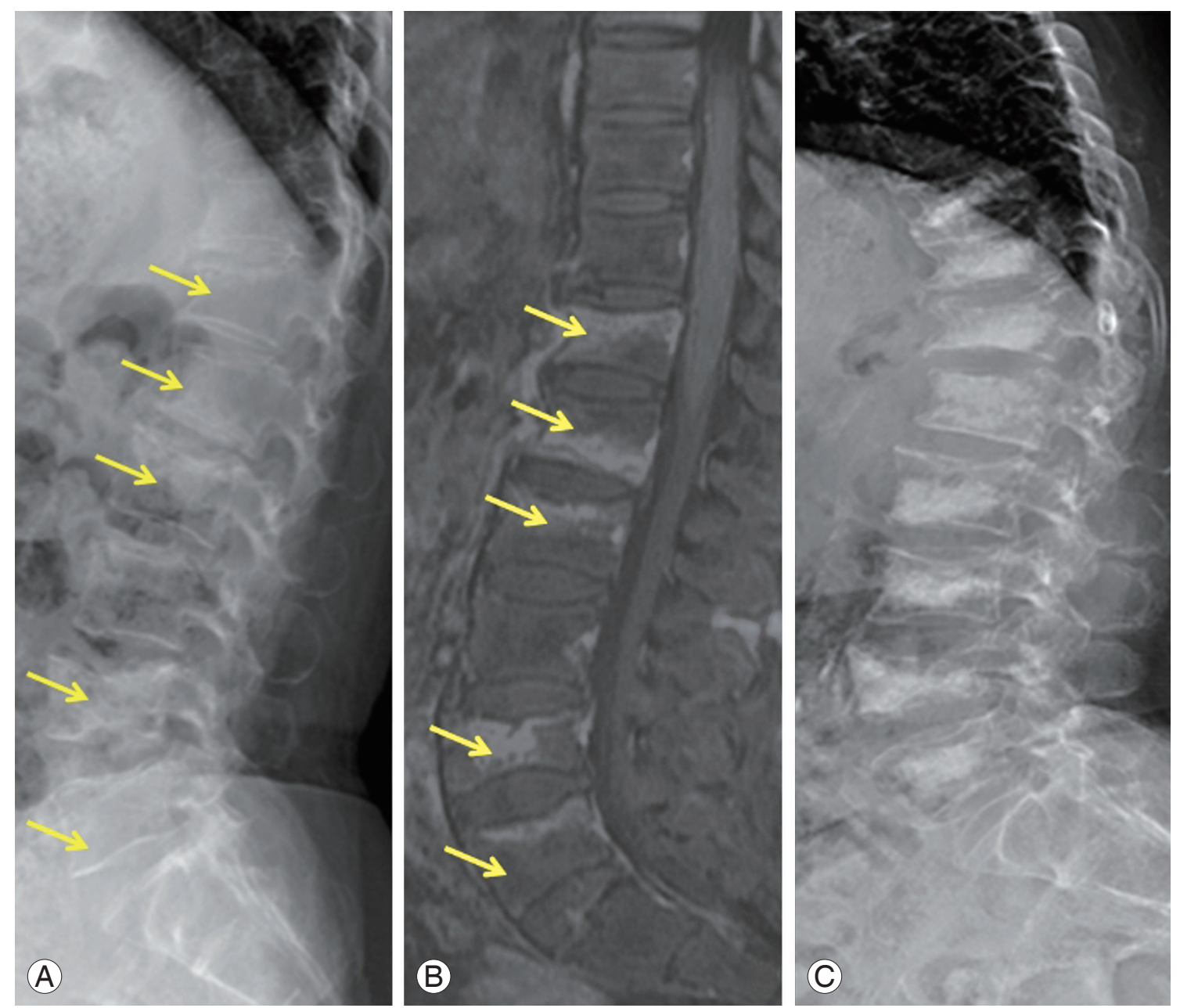

Fig. 2. (A, B) A 72-year-old female's simple lateral X-ray and magnetic resonance imaging showing multiple acute compression fracture. (C) Simple lateral radiograph at 6 months after vertebroplasty of multiple level. 
body during vertebroplasty in many patients.

Balloon kyphoplasty was recently developed to overcome these problems. Balloon kyphoplasty is effective in reducing pain, stabilizing the vertebral body, and correcting kyphotic deformity. During this procedure, the balloon is inserted in the vertebral body through a pedicle and inflated to lift the compressed vertebral body. The intervertebral space is then created and the balloon removed. This is followed by infusion of a substance such as bone cement at a lower pressure.

Balloon kyphoplasty is advantageous because it corrects the kyphotic angle and minimizes leakage of bone cement. It has recently been reported, however, that compression fractures of the adjacent vertebral bodies can occur after vertebroplasty. More attention should be paid to patients with secondary osteoporosis who present with a rapid progression of the fracture involving multilevel vertebrae during vertebroplasty. Methods such as vertebral reconstructive surgery, in which the multi- level vertebral compression fracture is reduced via the anterior or posterior approach, can also be considered. It is presumed, however, that even mechanical fixation will not provide the necessary fixation force due to severe osteoporosis. During vertebral reconstructive surgery, long, thick screws are used or, if applicable, bone cement is used concomitantly with screws. In addition, vertebral reconstructive surgery can be performed concomitantly with vertebroplasty. The tensile strength of the screws is increased with a hook. The most important consideration during vertebral reconstructive surgery is to disperse the tensile strength exerted on the screws through the fixation of multiple screws (Fig. 3). In these cases, however, there is a strong possibility that the scope and risks of surgery will increase. Accordingly, in patients with vertebral compression fractures involving multiple levels, the treatment goals of surgery are to achieve early ambulation and rehabilitation therapy or to maintain the balance of the vertebra on the sagittal or coronal plane. This may
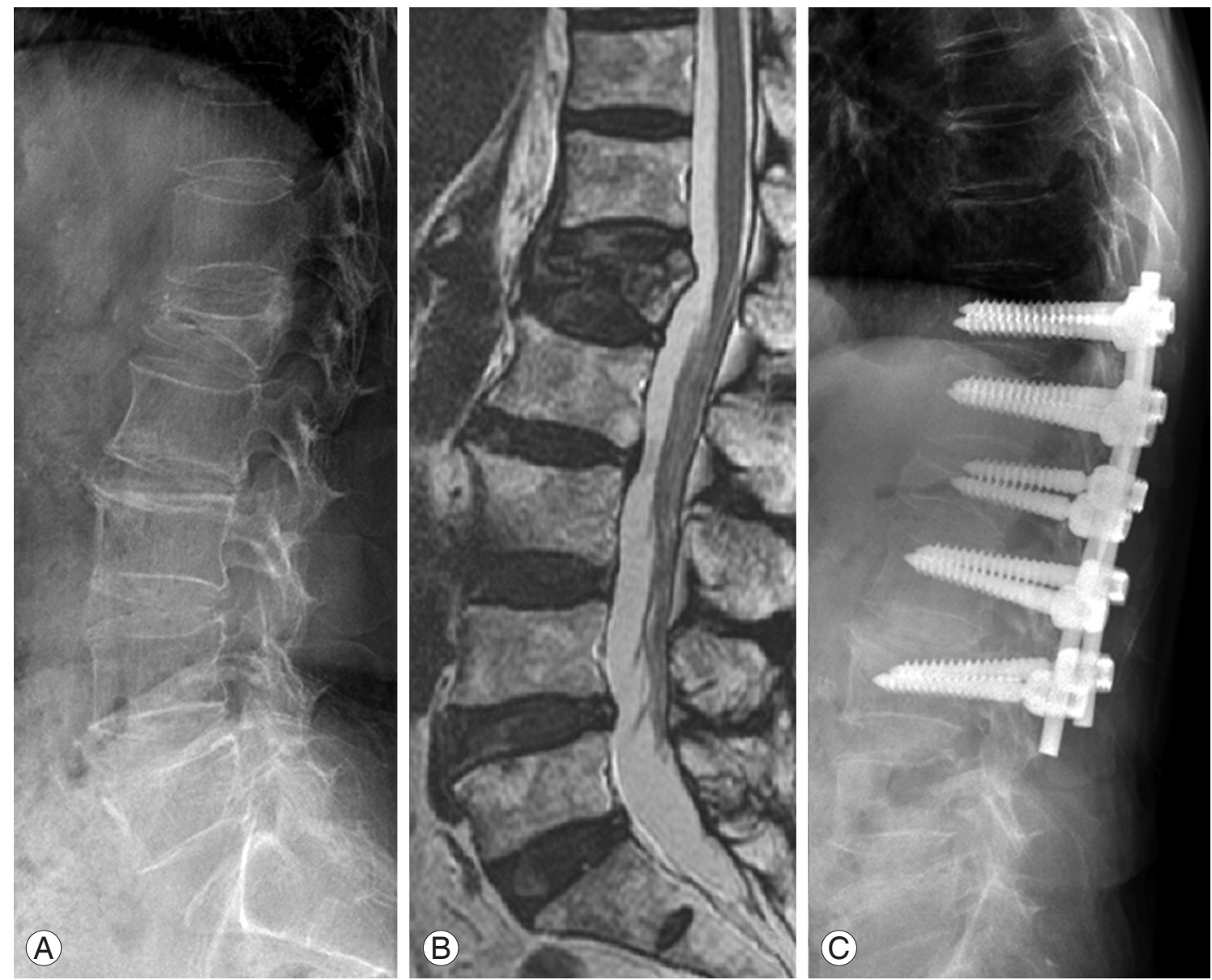

Fig. 3. (A, B) A 74-year-old female's simple X-ray and magnetic resonance imaging demonstrating acute osteoporotic compression fracture in L1 and resultant kyphosis. (C) Follow-up simple lateral X-ray at 6 month after the instrumentation. 
facilitate the determination of the optimal surgical modalities. It is of greater importance, however, to determine the optimal surgical modalities while considering the systemic status of patients.

\section{Other Conservative Treatments}

It is critically important to teach patients how to prevent osteoporosis during their daily lives. One-third of the elderly sustain a fall injury every year, and approximately $5 \%$ of them develop a fracture. This might be due to poor systemic status (e.g., muscle weakness, systemic malaise, impaired postural adjustment, decreased visual acuity, or decreased reflex), environmental factors in the home (e.g., poor lighting, slippery floors, or no walking assistance device in the bathroom), or systemic disease (e.g., cerebrovascular diseases, Parkinson's disease, arthritis, or cataracts).

In patients with osteoporosis, vertebral fracture may occur during simple activities, such as lifting or lowering an object or coughing. In patients with an established osteoporotic fracture, it is important to control pain and encourage early gait so that muscle exercise and stability of the fracture can be attained. Patients with acute lower back pain should be confined to bed for 2 to 3 days and accompanied by the use of analgesics, hot packs, massage, and lumbar orthosis. For the treatment of chronic pain, the back muscles should be strengthened with weightbearing activities including exercising and walking. Shoes or heels should be supported by a soft, elastic pad to prevent sliding. In addition, swimming and bicycling will help improve muscle strength and balance, which will help to reduce the occurrence of a fall injury. The use of a walking assistance device or orthosis can also help prevent patients with osteoporosis from sustaining a fall injury. Finally, patients with osteoporotic fractures may feel lethargy, anxiety, or depression due to the limitations in their daily lives and the alterations in their physical appearance. Therefore, the emotional support of family members is also essential for treating osteoporotic fractures.

\section{Conclusions}

To prevent multiple vertebral fractures, doctors must evaluate both the fractures and osteoporosis during treatment. This will help prevent the recurrence or aggravation of existing fractures. In addition, the occurrence of osteoporotic vertebral fractures is an irreversible process; these fractures cannot be healed. Therefore, pharmacological treatment should be considered to maintain bone quantity and prevent the occurrence of multiple vertebral fractures. The use of medications should be accompanied by regular medical follow-up. In these patients, changes in the BMD of the trabecular bone, as well as BMD itself, should be monitored. Moreover, methods for examining the microstructure of trabecular bone should be considered. This should also be accompanied by the consideration of active treatment for patients with multiple compression fractures.

\section{Conflict of Interest}

No potential conflict of interest relevant to this article was reported.

\section{References}

1. NIH Consensus Development Panel on Osteoporosis Prevention, Diagnosis, and Therapy. Osteoporosis prevention, diagnosis, and therapy. JAMA 2001;285:785-95.

2. Schuit SC, van der Klift M, Weel AE, et al. Fracture incidence and association with bone mineral density in elderly men and women: the Rotterdam Study. Bone 2004;34:195-202.

3. Yoon HK, Park C, Jang S, Jang S, Lee YK, Ha YC. Incidence and mortality following hip fracture in Korea. J Korean Med Sci 2011;26:1087-92.

4. Ha YC, Cho MR, Park KH, Kim SY, Koo KH. Is surgery necessary for femoral insufficiency fractures after long-term bisphosphonate therapy? Clin Orthop Relat Res 2010;468:3393-8.

5. Kim KC, Ha YC, Kim TY, Choi JA, Koo KH. Initially missed occult fractures of the proximal femur in elderly patients: implications for need of operation and their morbidity. Arch Orthop Trauma Surg 2010;130:915-20.

6. Weinstein RS, Jilka RL, Parfitt AM, Manolagas SC. Inhibition of osteoblastogenesis and promotion of apoptosis of osteoblasts and osteocytes by glucocorticoids. Potential mechanisms of their deleterious effects on bone. J Clin Invest 1998;102:274-82.

7. Dempster DW, Cosman F, Parisien M, Shen V, Lindsay R. Anabolic actions of parathyroid hormone on 
bone. Endocr Rev 1993;14:690-709.

8. Choi WH, Kim SY, Kim TH. Vertebral fracture in long term steroid dependent patients. Korean J Bone Metab 1994;1:224-29.

9. Holick MF. Vitamin D deficiency. N Engl J Med 2007;357:266-81.

10. Wolff AE, Jones AN, Hansen KE. Vitamin D and musculoskeletal health. Nat Clin Pract Rheumatol 2008;4:580-8.

11. Holick MF. High prevalence of vitamin D inadequacy and implications for health. Mayo Clin Proc 2006;81:353-73.

12. Neer RM, Arnaud CD, Zanchetta JR, et al. Effect of parathyroid hormone (1-34) on fractures and bone mineral density in postmenopausal women with osteoporosis. N Engl J Med 2001;344:1434-41.

13. Orwoll ES, Scheele WH, Paul S, et al. The effect of teriparatide [human parathyroid hormone (1-34)] therapy on bone density in men with osteoporosis. J Bone Miner Res 2003;18:9-17.

14. Body JJ, Gaich GA, Scheele WH, et al. A randomized double-blind trial to compare the efficacy of teriparatide [recombinant human parathyroid hormone (1-34)] with alendronate in postmenopausal women with osteoporosis. J Clin Endocrinol Metab 2002;87:4528-35.

15. Canalis E, Giustina A, Bilezikian JP. Mechanisms of anabolic therapies for osteoporosis. N Engl J Med 2007;357:905-16.

16. Jolette J, Wilker CE, Smith SY, et al. Defining a noncarcinogenic dose of recombinant human parathyroid hormone 1-84 in a 2-year study in Fischer 344 rats. Toxicol Pathol 2006;34:929-40. 